\title{
Calibration of Spectral Imaging Devices With Oxygenation-Controlled Phantoms: Introducing a Simple Gel-Based Hemoglobin Model
}

\author{
V. N. Du Le 1*, Myla Manser ${ }^{2}$, Sunny Gurm ${ }^{3}$, Ben Wagner ${ }^{3}$, Joseph E. Hayward ${ }^{4}$ and \\ Qiyin Fang ${ }^{2,4}$
}

${ }^{1}$ Department of Biomedical Engineering, University of California, Davis, Davis, CA, United States, ${ }^{2}$ Engineering Physics, McMaster University, Hamilton, ON, Canada, ${ }^{3}$ Christie Medical Holdings, Kitchener, ON, Canada, ${ }^{4}$ School of Biomedical Engineering, McMaster University, Hamilton, ON, Canada

OPEN ACCESS

Edited by:

Zhen Cheng,

Stanford University, United States

Reviewed by:

Roberta Frass-Kriegl,

Medical University of Vienna, Austria

Ashwin B. Parthasarathy

University of South Florida,

United States

*Correspondence:

V. N. Du Le

vdule@ucdavis.edu

Specialty section: This article was submitted to Medical Physics and Imaging,

a section of the journal

Frontiers in Physics

Received: 31 July 2019 Accepted: 04 November 2019 Published: 21 November 2019

Citation:

Le VND, Manser M, Gurm S, Wagner B, Hayward JE and Fang $Q$ (2019) Calibration of Spectral Imaging Devices With Oxygenation-Controlled Phantoms: Introducing a Simple Gel-Based Hemoglobin Model. Front. Phys. 7:192

doi: 10.3389/fphy.2019.00192
An optical phantom model with stable oxygen saturation (StO2) and optical properties (OPs) is the key for quick and accurate calibration of spectral imaging devices. Toward this goal, we have developed gel-based phantoms using Agarose, polystyrene microsphere, purified hemoglobin, and yeast. Theoretical values of OPs were calculated by applying Beer Lambert law and Mie theory whereas a robust fitting algorithm was used to extract $\mathrm{StO} 2$. Stability of OPs and $\mathrm{StO} 2$ in the phantoms was validated experimentally over a period of 2 weeks and the effect of pre-heating at $65^{\circ} \mathrm{C}$ degrees on $\mathrm{StO} 2$ was observed. Multi-spectral imaging $(750,780,850$, and $945 \mathrm{~nm})$ of the phantoms were also performed on the FDA-approved commercial system to demonstrate the utility of the phantoms.

Keywords: multi-spectral imaging (MSI), hemoglobin, optical properties, oxygen saturation, optical phantom, scattering, polystyrene microsphere

\section{INTRODUCTION}

Optical phantoms play an important role in calibrating and evaluating the performance optical spectroscopy and spectral imaging systems. Many different models of liquid-based, gelatin-based, and solid optical phantoms have been developed for this purpose [1]. In our previous works, different materials were selected to fabricate liquid-based phantoms for study of diffuse reflectance spectroscopy and fluorescence spectroscopy $[2,3]$. In general, desirable characteristics of optical phantoms are well-controlled optical properties, and the ability to mimic the spectral shape of tissue scattering and absorption. While hemoglobin $(\mathrm{Hb})$ absorption represents a key source of crosstalk in optical properties extraction and distortion in collected signal $[3,4]$, its optical signatures over a wide spectral range are the keys to non-invasive assessment of tissue oxygenation with multi-spectral imaging (MSI) systems [5, 6]. Although powdered Hb (i.e., H0267, Sigma-Aldrich) has been widely used to fabricate phantoms for calibration of fiber-optics-based techniques [2$4,7]$, it is not an ideal material for varying and accurately controlling of oxygen saturation. This is due to the autoxidation of the present heme group leading to the formation of the ferric derivative (methemoglobin) over time in addition to the already existed small amount of methemoglobin in the powder. Furthermore, powdered $\mathrm{Hb}$ is expensive whereas wide field near-infrared imaging technique requires large-volume phantoms to meet semi-infinite condition for photon propagation. Thus, the cost of phantom production significantly increases in such cases.

Meanwhile, although real human blood can be a good alternative to study oxygenation, it requires complicated sample preparation and storage. For example, whole blood needs to be in a pH 
and temperature controlled medium, and requires oxygenators and blood analyzers to change and test oxygen levels $[8,9]$. Another alternative is to use non-biological dye to mimic the absorption of whole blood in its deoxygenated state [10]. However, such approach is limited to a few near infrared wavelengths.

The purpose of this study is to develop a simple gelatin-based phantom model for quick calibration of spectral imaging systems at a broad-band spectrum. These phantoms should exhibit the spectral absorption characteristics of $\mathrm{Hb}$ and can be used to assess oxygen information. Beer Lambert law and Mie theory were used to guide the design of the phantom and the resulting optical properties and $\mathrm{StO} 2$ were validated experimentally. Measurements of the phantoms are also performed on a FDAapproved multi-spectral imaging (MSI) system.

\section{METHODS}

\section{Optical Phantoms}

To simulate tissue scattering, polystyrene microspheres with diameter of $1 \mu \mathrm{m}$ (07310-15, Polysciences Inc., PA) were used. To simulate tissue absorption, purified human hemoglobin (Multi4 CO-Oximeter Control Level 2, Instrumentation Laboratory Co., Bedford, MA) was used. The stock concentration of the $\mathrm{Hb}$ solution is $150 \mathrm{mg} / \mathrm{ml}$ with an average oxygen saturation (StO2) value of $96.9 \%[11,12]$. Polystyrene microspheres were selected as scatterers because of their stability over time, and of their well-controlled size and index of refraction [1]. To vary StO2 value, different yeast concentrations (Y0375, SigmaAldrich, Missouri) were added to the Hb solution [13]. As shown in Table 1, two groups of phantoms were created: control (Ctrl.) group without microspheres for transmission measurement and an experimental (Expt.) group with microspheres for MSI measurement.

Concentration of $\mathrm{Hb}$ was kept constant at $7 \mathrm{mg} / \mathrm{ml}$ in all phantoms, and the concentration of microspheres was $0.73 \%$ $w / v$ in the experimental group. Hemoglobin concentration of $7 \mathrm{mg} / \mathrm{ml}$ was chosen to obtain enough reflection with the multi-spectral imaging system. This concentration is similar to previous studies [4, 13]. Furthermore, our goal is to measure $\mathrm{StO} 2$, and total $\mathrm{Hb}$ concentration in the phantom doesn't affect

TABLE 1 | List of optical phantoms and their compositions.

\begin{tabular}{lcccc}
\hline Phantom label & $\begin{array}{c}\text { Microsphere } \\
\text { (w/v) }\end{array}$ & $\begin{array}{c}\text { Agarose } \\
\text { (w/v) }\end{array}$ & $\begin{array}{c}\text { Hemoglobin } \\
\text { (mg/ml) }\end{array}$ & $\begin{array}{c}\text { Yeast } \\
(\mathbf{w} / \mathbf{v})\end{array}$ \\
\hline Ctrl.HbY0 & 0 & $0.75 \%$ & 7 & 0 \\
Ctrl.HbY5 & 0 & $0.75 \%$ & 7 & $5 \%$ \\
Ctrl.HbY10 & 0 & $0.75 \%$ & 7 & $10 \%$ \\
Ctrl.HbY15 & 0 & $0.75 \%$ & 7 & $15 \%$ \\
Expt.HbY5 & $0.73 \%$ & $0.75 \%$ & 7 & $5 \%$ \\
Expt.HbY10 & $0.73 \%$ & $0.75 \%$ & 7 & $10 \%$ \\
Expt.HbY15 & $0.73 \%$ & $0.75 \%$ & 7 & $15 \%$
\end{tabular}

StO2 level. The concentrations of yeast in the final volume were 5,10 , and $15 \% w / v$, and were used to create phantoms with approximate $\mathrm{StO} 2$ values of 90,85 , and $50 \%$, respectively. Polystyrene microsphere has higher density than water, causing it to sink to the bottom of a liquid-based phantom after a period of time. This problem can be avoided using gel-based phantoms. The Agarose PCR grade (R9012HR, Cedarlane Laboratories, Burlington, ON) was selected to fabricate gel-based phantom because of its high gel strength and 100\% transmittance at gel concentration of $0.75 \% \mathrm{w} / \mathrm{v}$.

The use of yeast to reduce $\mathrm{StO} 2$ was previously described by Jang et al. [13]. The preparation of yeast in the current study is also similar so that yeast was completely dissolved in water at $65^{\circ} \mathrm{C}$ before mixing with hemoglobin solution. The main differences are: Agar gel is used in this study and the curing process is not required, and microsphere is used in this study as scatterers instead of Intralipid. The concentrated $4 \% w / v$ gel solution was previously prepared by mixing $0.78 \mathrm{~g}$ of Agar in distilled water at $65^{\circ} \mathrm{C}$, at which Agar is completely dissolved in $2 \mathrm{~h}$. The concentrated Agar solution was then added to the freshly made mixture of hemoglobin, microsphere and yeast which was preheated at the same temperature in $30 \mathrm{~s}$. The final concentration of Agar in the phantom was $0.75 \%$ w/v (Table 1) which produces solid phantoms at room temperature. To prevent the formation of air bubbles, a $100 \mu$ l-pipette was used to slowly mix the solutions.

In all phantoms, the total absorption coefficient $\left(\mu_{\mathrm{a}}\right)$ is calculated by applying Beer-Lambert law to the measured transmission from a spectrophotometer (Ultraspec 3,000, Pharmacia Biotech Inc., NJ) whereas $\mu_{\mathrm{s}}$ ' is calculated by applying Mie theory to 1 - $\mu \mathrm{m}$-diameter polystyrene microsphere $[2,3,7]$. The final volume of each phantom was $15 \mathrm{ml}$, and all phantoms were stored at $4^{\circ} \mathrm{C}$ degrees. In addition, a fitting approach was

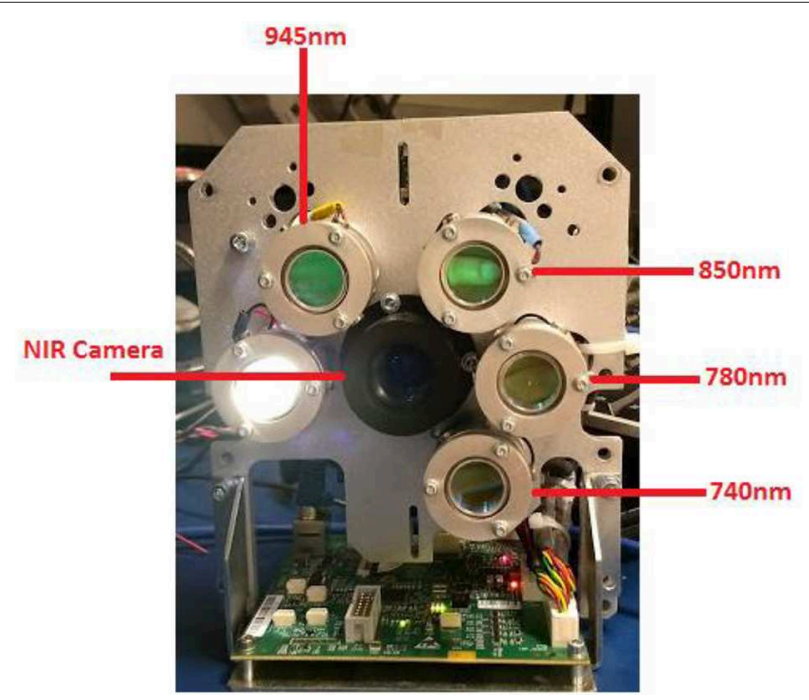

FIGURE 1 | Image of the commercial multispectral imaging system (Presygen $\left.^{\mathrm{TM}} / \mathrm{si}-1\right)$. 

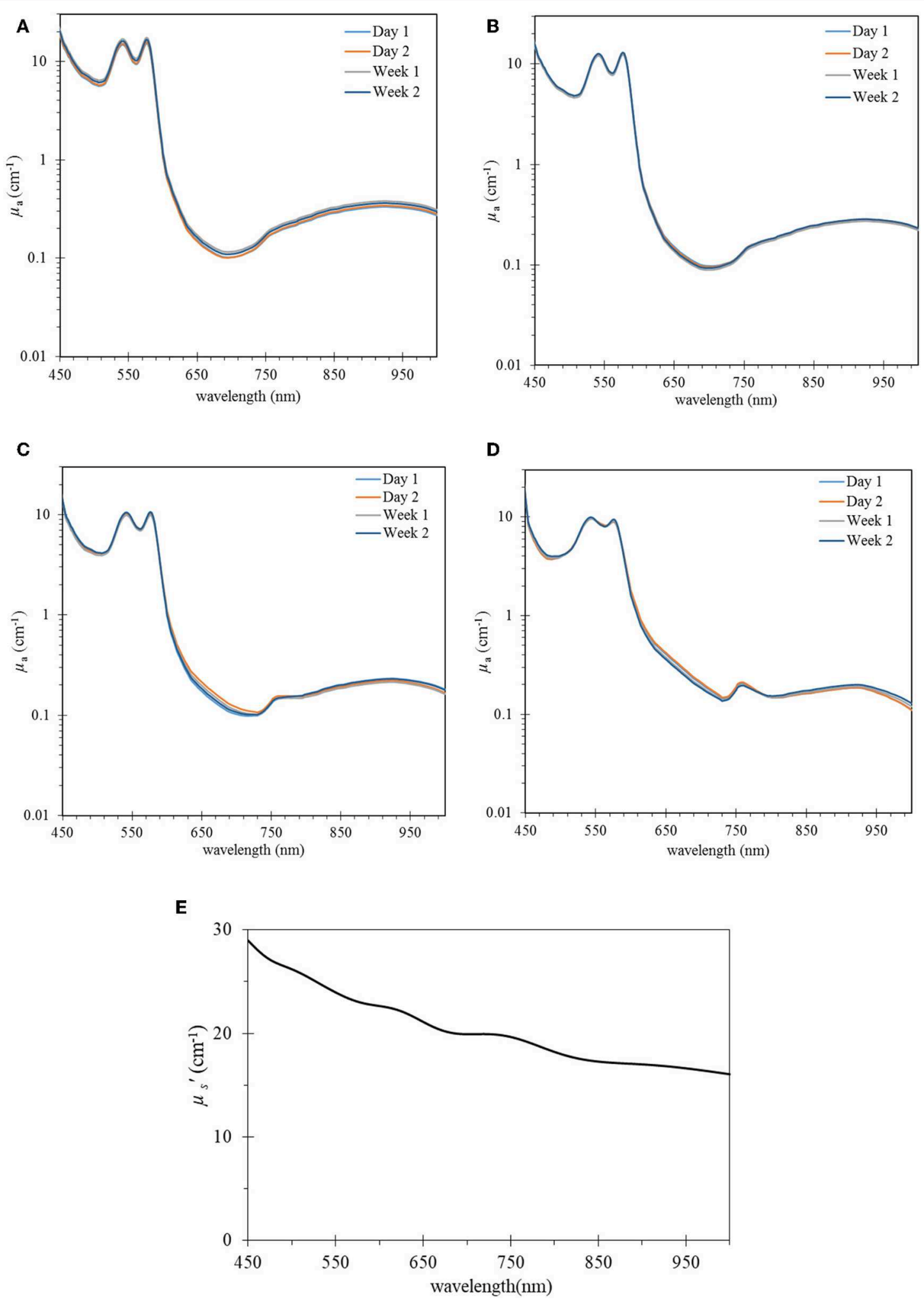

FIGURE 2 | $\mu_{\mathrm{a}}$ spectra over time of four control phantoms (A) hemoglobin without yeast (Ctr.HbY0), (B) with yeast 5\% (Ctr.HbY5), (C) with yeast 10\% (Ctr.HbY10), (D) with yeast 15\% (Ctr.HbY15). Hemoglobin concentration in all phantoms was kept constant at $7 \mathrm{mg} / \mathrm{ml}$ and $\mu_{\mathrm{s}}{ }^{\prime}$ spectrum of baseline microsphere $0.73 \%$ w/v (E). 
applied to fit the total absorption coefficient, $\mu_{\mathrm{a}}$ and to obtain the oxygen saturation value $[4,7]$, such that:

$$
\mu_{\mathrm{a}}(\lambda)=\ln (10) \cdot f_{1} \cdot\left[f_{2} \cdot \varepsilon_{\mathrm{HbO} 2}(\lambda)+\left(1-f_{2}\right) \cdot \varepsilon_{\mathrm{Hb}}(\lambda)\right]+\mu_{\mathrm{a} \text {,yeast }}(1)
$$

In Equation (1), $\mu_{\mathrm{a} \text {,yeast }}\left(\mathrm{cm}^{-1}\right)$ is the absorption coefficient of yeast in Agar gel solution, $\lambda$ is wavelength, $f_{1}\left(\right.$ mol.liter $\left.^{-1}\right)$ is the total concentration of hemoglobin, $f_{2}$ (dimensionless) is oxygen saturation $(\mathrm{StO} 2)$, and $\varepsilon_{\mathrm{HbO} 2}\left(\mathrm{~cm}^{-1} \cdot \mathrm{M}^{-1}\right)$ and $\varepsilon_{\mathrm{Hb}}$ $\left(\mathrm{cm}^{-1} \cdot \mathrm{M}^{-1}\right)$ are molar extinction coefficients of oxygenated and deoxygenated hemoglobin, respectively. To obtain $f_{2}$, the least square fitting routine "fminsearch" in MATLAB ${ }^{\circledR}$ was used to fit the broadband measurement of $\mu_{\mathrm{a}}$ and $\mu_{\mathrm{a} \text {,yeast }}$ into Equation (1). This optimization method is based on the Nelder-Mead simplex algorithm and has been widely used for spectral analysis [1416]. The volume of the phantoms satisfied semi-infinite condition for photon migration. This can be estimated by comparing the phantom thickness to the optical penetration depth obtained from diffusion theory.

\section{Multi-Spectral Imaging (MSI) System}

A commercial multi-spectral imaging system (Presygen ${ }^{\mathrm{TM}} / \mathrm{si}-1$, Christie Digital Systems, Kitchener, ON) was used to estimate $\mathrm{StO} 2$ in the phantoms. The system includes four near Infrared (NIR) LEDs with illumination wavelengths at 740, 780, 850, and $945 \mathrm{~nm}( \pm 10 \mathrm{~nm})$, and a NIR camera mounted on an imaging head (Figure 1). Each LED is turned on sequentially, and an image is captured using custom controller software. A
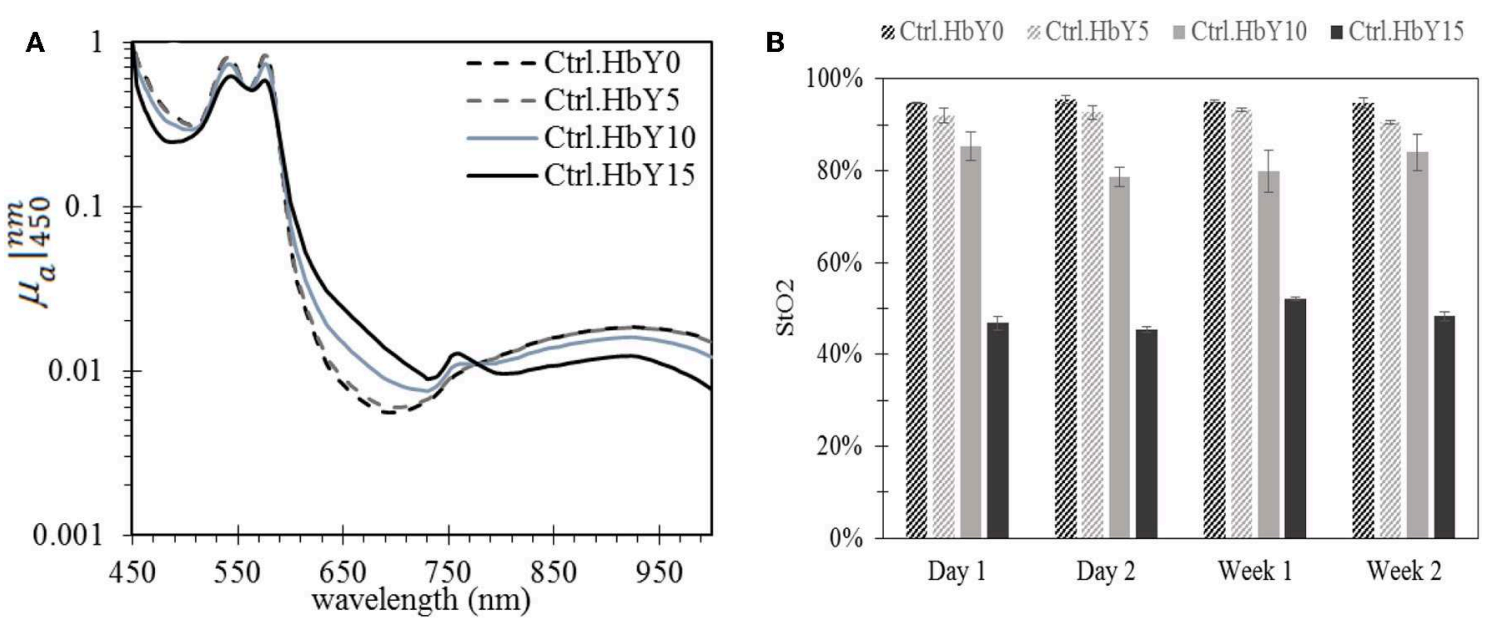

FIGURE 3 | Oxygen saturation affects hemoglobin absorption spectrum: (A) Normalized $\mu_{\mathrm{a}}$ spectra with respect to $\mu_{\mathrm{a}}$ at $450 \mathrm{~nm}$ and (B) oxygen saturation (StO2) over time in four control phantoms: hemoglobin without yeast (Ctr.HbY0), with yeast 5\% (Ctr.HbY5), 10\% (Ctr.HbY10), and 15\% (Ctr.HbY15). Hemoglobin concentration in all phantoms was kept constant at $7 \mathrm{mg} / \mathrm{ml}$.
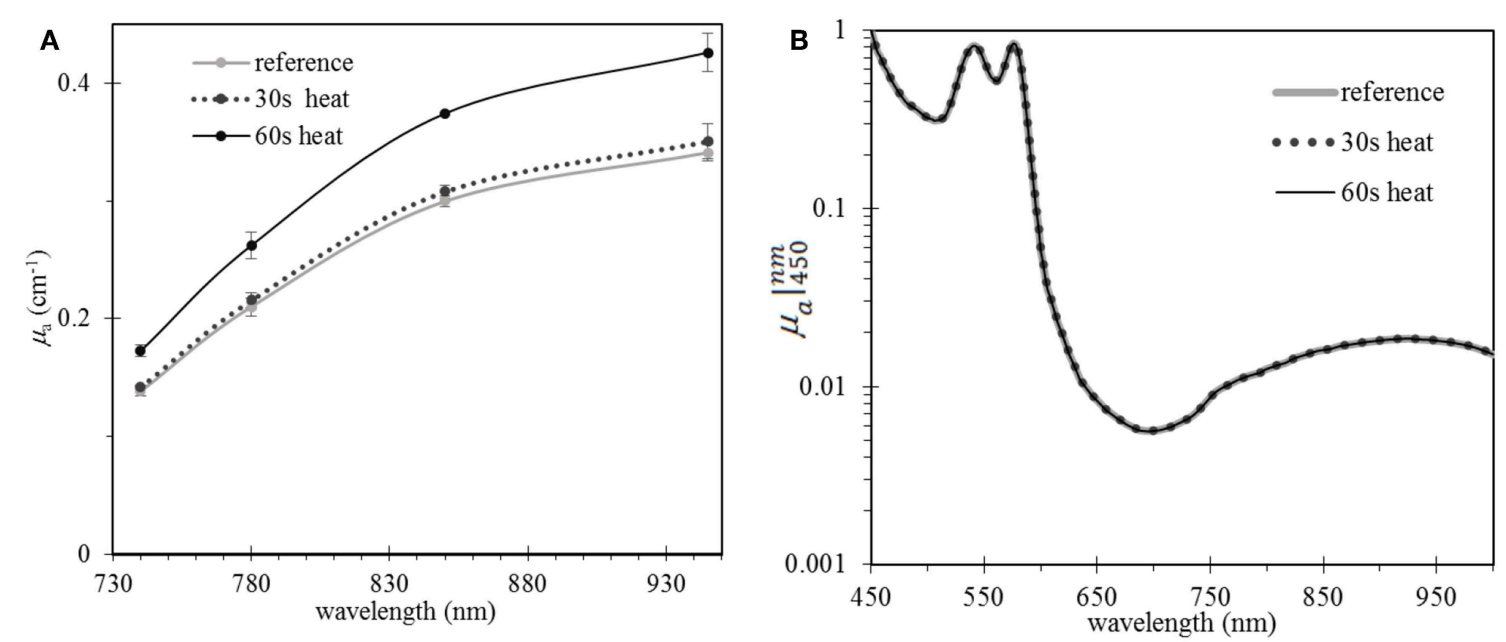

FIGURE 4 | Examples of heating can increase hemoglobin concentration but do not affect the shape of absorption spectrum: Effect of prolonged heating time up to 1 min on (A) $\mu_{\mathrm{a}}$ values at four wavelengths, and (B) $\mu_{\mathrm{a}}$ spectral shape. Data from phantom Ctrl. HbYO was presented. Heating temperature is kept at $65^{\circ} \mathrm{C}$ degrees. The "reference" curve represents a liquid phantom that was not pre-heated. 
background image is captured where none of the LEDs are turned on, and is subtracted from NIR images to account for ambient light. The MSI system utilizes a previously developed scatter model to calculate $\mathrm{StO} 2$ values of the experimental phantoms [17]. The calculated $\mathrm{StO} 2$ values were compared to those of control phantoms measured with spectrophotometer.

\section{RESULTS AND DISCUSSION}

Figure 2 shows $\mu_{\mathrm{a}}$ spectra of four control phantoms over period of 2 weeks whereas (Figure 3) compares the normalized $\mu_{\mathrm{a}}$ spectra of four phantoms in the wavelength region of 450$1,000 \mathrm{~nm}$. In general, the characteristic absorption peaks at 540 and $580 \mathrm{~nm}$ were observed in all phantoms (Figures 2, 3A), and no significant change in $\mu_{\mathrm{a}}$ spectra was observed in the period of 2 weeks in all phantoms (Figure 2). Figure 2E shows the reduced scattering. In addition, a noticeable $\mu_{\mathrm{a}}$ peak at the $750 \mathrm{~nm}$ region and a shift upward in $\mu_{\mathrm{a}}$ spectrum in the $650 \mathrm{~nm}$ region were observed in phantom with high yeast concentrations of $15 \%$ (Figure 3A). These are well-known features when oxy-Hb changes into deoxy- $\mathrm{Hb}[18,19]$. Figure 3B shows $\mathrm{StO} 2$ values in four control phantoms over 2 weeks. In each phantom, the percentage difference between $\mathrm{StO} 2$ value in day 1 and week 2 was within $6 \%$. On average, the calculated $\mathrm{StO} 2$ value was $94.6 \% \pm 2.7 \%, 92.2 \% \pm 3.2 \%, 81.93 \pm 5.2 \%, 48.2 \pm 5.9 \%$ for phantom Ctrl.HbY0, CTrl.HbY5, Ctrl.HbY10, and Ctrl.HbY15, respectively. Error bars in Figure $\mathbf{3 B}$ represent the standard deviation for measurements of three similar phantoms that were fabricated separately but with the same yeast concentration (and everything else). Such averaging accounts for errors in fabrication procedure. Comparing week 1 and week 2, StO2 increases 5\% in Ctrl.HbY10, and decreases 3\% in Ctrl. HbY5, and $7 \%$ in Ctrl. HbY15.

All the gel phantoms were prepared at $65^{\circ} \mathrm{C}$ in $30 \mathrm{~s}$. Therefore, to determine the effect of heating duration on $\mathrm{StO} 2$, a set of Ctrl.HbY0 phantoms were made, then heated at $65^{\circ} \mathrm{C}$ in different time periods up to $1 \mathrm{~min}$. In general, prolonging heating time will increase absorption due to the loss of water through

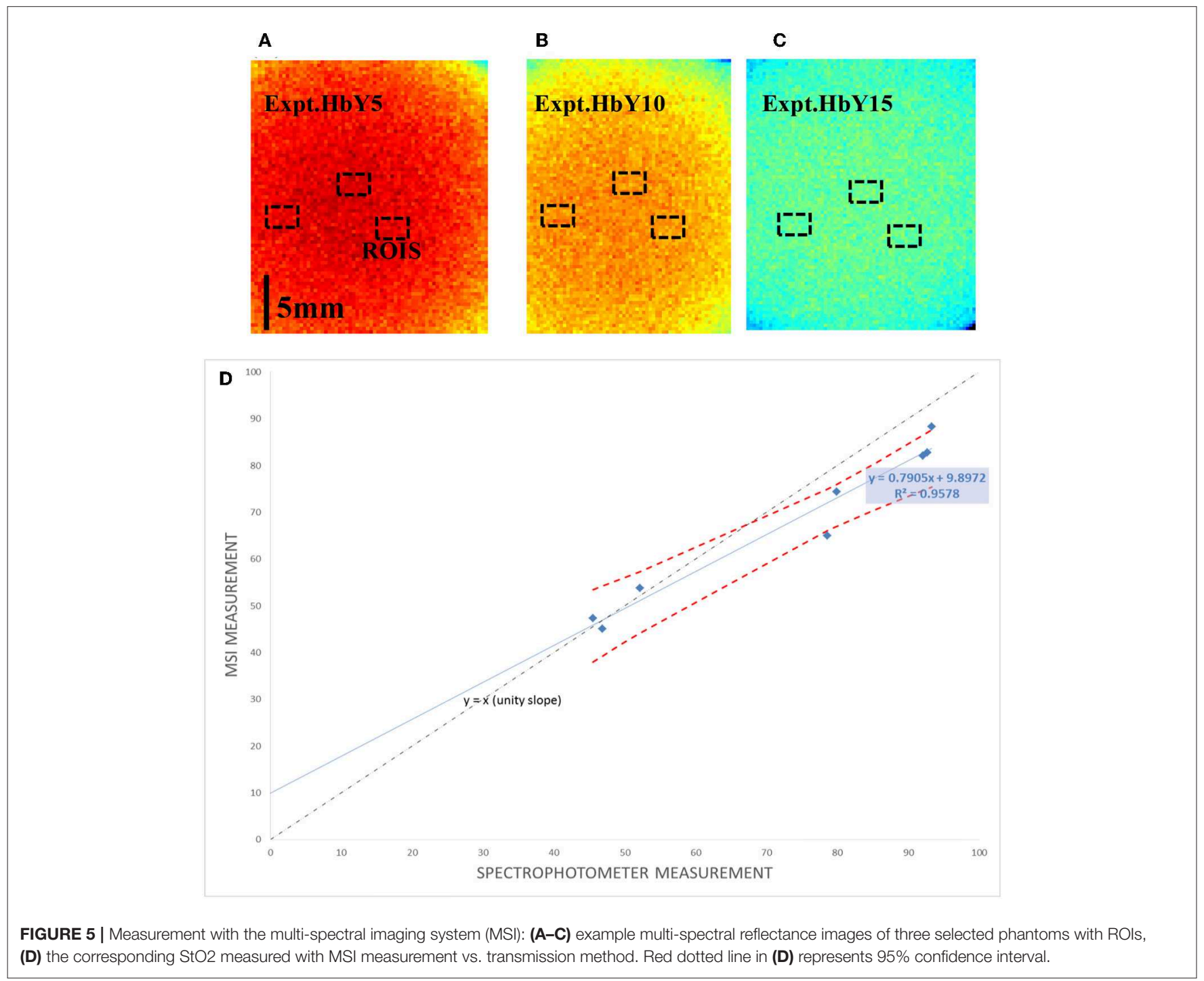


evaporation (Figure 4A). More specifically, $\mu_{\mathrm{a}}$ increases by 2.5 and $25.4 \%$ compared to the reference $\mu_{\mathrm{a}}$ if heating period is $30 \mathrm{~s}$ and $1 \mathrm{~min}$, respectively. However, $\mu_{\mathrm{a}}$ spectral shape was not affected by heating (Figure 4B). Because StO2 is determined by of the relative $\mu_{\mathrm{a}}$ values on the spectrum $[4,20]$, the stability of $\mu_{\mathrm{a}}$ spectral shape ensures stability of the $\mathrm{StO} 2$ value. In these phantoms, the average $\mathrm{StO} 2$ value was $96 \pm 3 \%$.

The MSI system was used to capture the experimental phantoms so that 2D spatial images of StO2 were generated for each phantom over course of 1 week to account for day to day variations and aging. Each phantom was imaged at the date of manufacture, day 3, and day 7. For each phantom, averaging over three regions of interests (ROIs) was performed to account for spatial variation. (Figures 5A-C). As shown in Figure 5D, a good agreement between spectrophotometer measurements and MSI measurement is obtained with an averaged percentage difference of $8 \%$.

In this paper, the phantoms are selected so that the reflectance signal is within the detection capability of the commercial multispectral imaging system. Currently, this system can extract useful information from highly turbid media with minimal absorption (where diffusion theory is valid and where signal to noise ratio is high enough). A further increase in yeast concentration and/or hemoglobin concentration can increase the total absorption coefficient, reducing the accuracy of the Presygen system, and hence is avoided. Meanwhile, vaporization is significant with prolonged aging, causing an increase in absorber concentration, thus increasing total absorption of the phantoms. Therefore, longer aging is not considered when using the Presygen system. Nevertheless, the selected ranges of optical properties and oxygen saturation in the current phantom model are within literature for tissues and optical phantoms [7, 21, 22].

\section{CONCLUSIONS}

In this study, we have demonstrated a gel-based optical phantom model with controlled oxygenation that can be used for quick

\section{REFERENCES}

1. Pogue BW, Patterson MS. Review of tissue simulating phantoms for optical spectroscopy, imaging and dosimetry. J Biomed Opt. (2006) 11:041102. doi: $10.1117 / 1.2335429$

2. Du Le VN, Nie Z, Hayward JE, Farrell TJ, Fang Q. Measurements of extrinsic fluorescence in Intralipid and polystyrene microspheres. Biomed Opt Exp. (2014) 5:2726-35. doi: 10.1364/BOE.5.002726

3. Du Le VN, Patterson MS, Farrell TJ, Hayward JE, Fang Q. Experimental recovery of intrinsic fluorescence and fluorophore concentration in the presence of hemoglobin: spectral effect of scattering and absorption on fluorescence. J Biomed Opt. (2015) 20:127003. doi: 10.1117/1.JBO.20.12.127003

4. Wang Q, Le D, Ramella-Roman J, Pfefer J. Broadband ultravioletvisible optical property measurement in layered turbid media. Biomed Opt Exp. (2012) 3:1226-40. doi: 10.1364/BOE.3. 001226

5. Li Q, He X, Wang Y, Liu H, Xu D, Guo F. Review of spectral imaging technology in biomedical engineering: achievements and challenges. J Biomed Opt. (2013) 18:100901. doi: 10.1117/1.JBO.18.10100901 calibration of spectral imaging devices without the use of whole blood or powdered hemoglobin, and that yeast can be used to decrease the oxygen saturation of a commercial blood product that is fully oxygenated and is more cost effective than powdered hemoglobin. In addition, although increasing yeast concentration decreases oxygen saturation of the phantom, the phenomenon does not occur linearly. Also, a short period of heating can vary the concentration of hemoglobin but does not have major effect on the phantom oxygen saturation. In other words, heating changes the magnitude of absorption but does not change its spectral shape. Specifically, $\mu_{\mathrm{a}}$ increases about $2.5 \%$ due to heating duration of $30 \mathrm{~s}$ at $65^{\circ} \mathrm{C}$ whereas the absorption spectral shape of the selected hemoglobin was not affected. In addition, the current gel model is able to produce the consistent results in the period of 2 weeks with a relative uncertainty of $<6 \%$. The phantom is low cost and simple to make with excellent repeatability, which is critical for its practical application. Therefore, the current gel phantoms have desirable characteristics for calibrating multi-spectral imaging systems, including cost effectiveness, well-controlled optical properties and oxygen saturation, and the ability to retain stability over an adequate period of time.

\section{DATA AVAILABILITY STATEMENT}

The datasets generated for this study are available on request to the corresponding author.

\section{AUTHOR CONTRIBUTIONS}

VL supervised the production of all optical phantoms, applied theories and developed algorithms to control the optical properties, and oxygen saturation in the phantom. MM assisted with data measurement and phantom fabrication. SG and BW measured the phantoms with the spectral imaging system. JH and QF advised the progress of the study.
6. Xu RX, Allen DW, Huang J, Gnyawali S, Melvin J, Elgharably H, et al. Developing digital tissue phantoms for hyperspectral imaging of ischemic wounds. Biomed Opt Exp. (2012) 3:1433-45. doi: 10.1364/BOE.3.001433

7. Du Le VN, Provias J, Murty N, Patterson MS, Nie Z, Hayward JE, et al. Dualmodality optical biopsy of glioblastomas multiforme with diffuse reflectance and fluorescence: ex vivo retrieval of optical properties. J Biomed Opt. (2017) 22:027002. doi: 10.1117/1.JBO.22.2.027002

8. Faber DJ, Aalders MC, Mik EG, Hooper BA, van Gemert MJ, van Leeuwen TG. Oxygen saturation-dependent absorption and scattering of blood. Phys Rev Lett. (2004) 93:028102. doi: 10.1103/PhysRevLett.93.028102

9. Wang PJ, Li WC, Xi GM, Wang HQ, Zhang ZH, Yao BC, et al. Biomechanical study of hepatic portal vein in humans and pigs and its value in liver transplantation. Transplant Proc. (2009) 41:1906-10. doi: 10.1016/j.transproceed.2008.10.097

10. Akl TJ, Long R, McShane MJ, Ericson MN, Wilson MA, Coté GL. Optimizing probe design for an implantable perfusion and oxygenation sensor. Biomed Opt Exp. (2011) 2:2096-109. doi: 10.1364/BOE.2.002096

11. Steinke JM, Shepherd AP. Effects of temperature on optical absorbance spectra of oxy-, carboxy-, and deoxyhemoglobin. Clin Chem. (1992) 38:1360-4. 
12. Bosschaart N, Edelman GJ, Aalders MC, van Leeuwen TG, Faber DJ. A literature review and novel theoretical approach on the optical properties of whole blood. Lasers Med Sci. (2014) 29:453-79. doi: 10.1007/s10103-013-1446-7

13. Jang $\mathrm{H}$, Pfefer TJ, Chen Y. Solid hemoglobin-polymer phantoms for evaluation of biophotonic systems. Opt Lett. (2015) 40:4321-4. doi: 10.1364/OL.40.004321

14. Jacques SL, Samatham R, Choudhury N. Rapid spectral analysis for spectral imaging. Biomed Opt Exp. (2010) 1:157-64. doi: 10.1364/BOE.1.000157

15. Twardowski MS, Boss ES, Sullivan JM, Donaghay PL. Modeling the spectral shape of absorption by chromophoric dissolved organic matter. Mar Chem. (2004) 89:69-88. doi: 10.1016/j.marchem.2004.02.008

16. Zhong X, Wen X, Zhu D. Lookup-table-based inverse model for human skin reflectance spectroscopy: two-layered Monte Carlo simulations and experiments. Opt Exp. (2014) 22:1852-64. doi: 10.1364/OE.22.001852

17. Savelieva TA, Stratonnikov AA, Loschenov V. "Multi-spectral imaging of oxygen saturation," in Proceedings of SPIE - The International Society for Optical Engineering, 7022. doi: 10.1117/12.803905

18. Prahl SA. Tabulated molar extinction coefficient for hemoglobin in water. In: Gratzer WB, and Kollias N, editors. Available online at http://omlc.org/ spectra/hemoglobin/summary.html (accessed March 4, 1998).

19. Prahl SA, van Gemert M, Welch AJ. Determining the optical properties of turbid media by using the adding-doubling method. Appl Opt. (1993) 32:559-68. doi: 10.1364/AO.32.000559
20. Seo I, Bargo PR, Kollias N. Simultaneous assessment of pulsating and total blood in inflammatory skin lesions using functional diffuse reflectance spectroscopy in the visible range. J Biomed Opt. (2010) 15:060507. doi: $10.1117 / 1.3524191$

21. Bargo PR, Prahl SA, Goodell TT, Sleven RA, Koval G, Blair G, et al. In vivo determination of optical properties of normal and tumor tissue with white light reflectance and an empirical light transport model during endoscopy. J Biomed Opt. (2005) 10:034018-1815. doi: 10.1117/1. 1921907

22. Jacques SL. Optical properties of biological tissues: a review. Phys Med Biol. (2013) 58:R37. doi: 10.1088/0031-9155/58/ 11/R37

Conflict of Interest: The authors declare that the research was conducted in the absence of any commercial or financial relationships that could be construed as a potential conflict of interest.

Copyright (C) 2019 Le, Manser, Gurm, Wagner, Hayward and Fang. This is an openaccess article distributed under the terms of the Creative Commons Attribution License (CC BY). The use, distribution or reproduction in other forums is permitted, provided the original author(s) and the copyright owner(s) are credited and that the original publication in this journal is cited, in accordance with accepted academic practice. No use, distribution or reproduction is permitted which does not comply with these terms. 